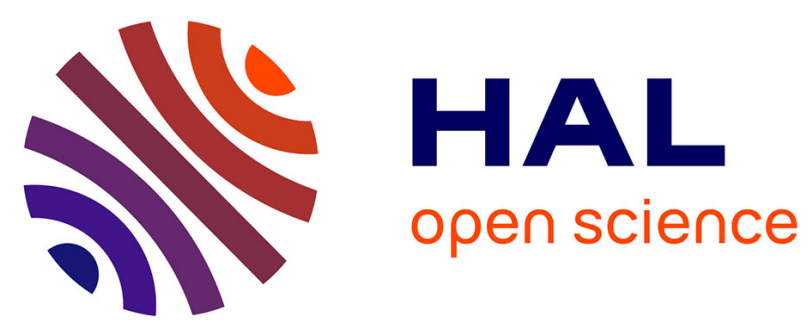

\title{
Dynamic imaging of a capillary-gravity wave in shallow water using amplitude variations of eigenbeams
}

\author{
Tobias van Baarsel, Philippe Roux, Jerome I. Mars, Julien Bonnel, M.
}

Arrigoni, Steven Kerampran, Barbara Nicolas

\section{- To cite this version:}

Tobias van Baarsel, Philippe Roux, Jerome I. Mars, Julien Bonnel, M. Arrigoni, et al.. Dynamic imaging of a capillary-gravity wave in shallow water using amplitude variations of eigenbeams. Journal of the Acoustical Society of America, 2019, 146 (5), pp.3353-3361. 10.1121/1.5132939 . hal-02380508

\section{HAL Id: hal-02380508 \\ https://hal.science/hal-02380508}

Submitted on 26 Nov 2019

HAL is a multi-disciplinary open access archive for the deposit and dissemination of scientific research documents, whether they are published or not. The documents may come from teaching and research institutions in France or abroad, or from public or private research centers.
L'archive ouverte pluridisciplinaire HAL, est destinée au dépôt et à la diffusion de documents scientifiques de niveau recherche, publiés ou non, émanant des établissements d'enseignement et de recherche français ou étrangers, des laboratoires publics ou privés. 


\title{
Dynamic imaging of a capillary-gravity wave in shallow water using amplitude variations of eigenbeams
}

\author{
Tobias van Baarsel, ${ }^{1, a)}$ Philippe Roux, ${ }^{1}$ Jérôme Igor Mars, ${ }^{2}$ Julien Bonnel, ${ }^{3}$ Michel Arrigoni, ${ }^{4}$ \\ Steven Kerampran, ${ }^{4}$ and Barbara Nicolas ${ }^{5}$ \\ ${ }^{1}$ Institut des Sciences de la Terre, Université Grenoble Alpes, 1381 rue de la Piscine, Grenoble 38041, France \\ ${ }^{2}$ Gipsa-lab, 11 rue des Mathématiques, Grenoble Campus BP46, F-38402 Saint Martin D' Héres, France \\ ${ }^{3}$ Woods Hole Oceanographic Institution, 266 Woods Hole Road, Woods Hole, Massachusetts 02543-1050, \\ USA \\ ${ }^{4}$ Ecole Nationale Supérieure de Techniques Avancées, 2 rue François Verny, 29806 Brest Cedex 09, France \\ ${ }^{5}$ Créatis, INSA Lyon, 7 avenue Jean Capelle, 69621 Villeurbanne, France
}

(Received 14 June 2019; revised 11 October 2019; accepted 15 October 2019; published online 15 November 2019)

\begin{abstract}
Dynamic acoustic imaging of a surface wave propagating at an air-water interface is a complex task that is investigated here at the laboratory scale through an ultrasonic experiment in a shallow water waveguide. Using a double beamforming algorithm between two source-receiver arrays, the authors isolate and identify each multi-reverberated eigenbeam that interacts with the air-water and bottom interfaces. The waveguide transfer matrix is recorded 100 times per second while a low-amplitude gravity wave is generated by laser-induced breakdown at the middle of the waveguide, just above the water surface. The controlled, and therefore repeatable, breakdown results in a blast wave that interacts with the air-water interface, which creates ripples at the surface that propagate in both directions. The amplitude perturbations of each ultrasonic eigenbeam are measured during the propagation of the gravity-capillary wave. Inversion of the surface deformation is performed from the amplitude variations of the eigenbeams using a diffraction-based sensitivity kernel approach. The accurate ultrasonic imaging of the displacement of the air-water interface is compared to simultaneous measurements with an optical camera, which provides independent validation.
\end{abstract}

(C) 2019 Acoustical Society of America. https://doi.org/10.1121/1.5132939

[SED]

Pages: 3353-3361

\section{INTRODUCTION}

Shallow waters are challenging environments for acoustic communication and target detection. As well as coastal currents, noise sources, and sound speed inhomogeneities, the rough surface and bottom cause reverberation and scattering, which are an issue for simple co-located source-receiver sonar systems (i.e., Kuperman and Lynch, 2004; Trevorrow, 1998). Some of these issues can be overcome in a bi-static sonar system (also called an acoustic barrier) when two source and receiver arrays face each other in shallow waters. In this configuration, detection of a target between the two arrays becomes a matter of detection of the fluctuations in the acoustic pressure field induced by the target through forward scattering. Unfortunately, these fluctuations are still polluted by perturbations of the oceanic environment, e.g., surface waves, tides, and bubbles. Therefore, the study of the effects of surface waves on the acoustic pressure field is crucial to enhance target detection in shallow waters (i.e., Marandet et al., 2011; Deane et al., 2012). Recent studies have dealt with statistical studies of the effects of the surface wave field on the acoustic wave field (Walstead and Deane, 2014), and the deterministic forward scatter of an acoustic wave on a travelling surface gravity wave (Deane et al., 2012). At the laboratory scale, the surface waves are governed by gravity-capillary forces, and Roux and Nicolas (2014) used the sensitivity kernel (SK)

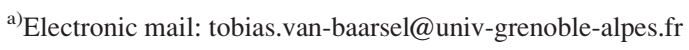

approach to successfully, although only qualitatively, image a travelling capillary-gravity surface wave between two ultrasonic transducer arrays. Furthermore, no independent information on the surface perturbation could be extracted from the experimental configuration.

This paper describes an experiment designed to allow quantitative inversion of two counter-propagating wave packets travelling at the surface of a shallow ultrasonic waveguide in a bi-static acoustic barrier configuration. By extending the experiment by Roux and Nicolas (2014) a step further, we show that unlike traditional tomography based on travel-time variations, we can now successfully image surface perturbations of the waveguide using only the amplitude information of the eigenbeams extracted between the source and receiver arrays. This means that no information about the absolute travel-time between sources and receivers is needed for this tomography technique. Also, the SK for each eigenbeam is now computed on the whole surface of the waveguide, instead of just as a one-dimensional line, as was the situation in previous studies.

In practice, the forward model is built using the SK approach with the assumption of a linear relation between the surface deformation and the ultrasonic eigenbeam amplitude perturbation in the first-order Born approximation. The surface perturbation extracted from the ultrasonic measurements inside the waveguide is then inverted using a classical maximum a priori (MAP) technique. We experimentally 
demonstrate that the SK inversion provides a quantitative measurement of the capillary-gravity wave dispersion that can be measured independently by optical means. A highspeed camera records the profile view of the waveguide, and directly measures the height and speed of the surface deformation. The cross-comparison of the ultrasonic and optical systems allows qualitative and quantitative independent validation of the inversion results, as well as open discussion on the limitations of the SK approach.

This paper is organized as follows, Section II describes the small-scale experiment as performed under laboratory conditions. Two different laser excitation levels are presented in two different experiments that show the upper and lower limits of the acoustic inversion. Section III explains the SK approach and the inversion procedure. Section IV presents the inversion results for the two laser excitations, and their validation using the camera-recorded data. Section $\mathrm{V}$ discusses the results and the methodology, and indicates their limitations.

\section{EXPERIMENTAL SETUP AND DATA ANALYSIS}

Following the methodology proposed by Roux and Nicolas (2014), a small-scale experiment is set up in a shallow water, 1-m-long, 55-mm-deep, ultrasonic waveguide, as illustrated in Fig. 1. This reproduces a coastal water environment at a scale of $1 / 1000$ (water depth, $\approx 55 \mathrm{~m}$; range, $\approx 1 \mathrm{~km}$ ). Two source-receiver vertical arrays that face each other record the ultrasonic response of the waveguide. The ultrasonic arrays comprise 64 transducers centered at $1 \mathrm{MHz}$, with an ultrasonic sampling rate of $10 \mathrm{MHz}$. The transducer dimensions are $0.75 \mathrm{~mm}$ along the vertical axis, and $12 \mathrm{~mm}$ along the transverse axis, which naturally creates a collimated beam in the waveguide axis direction. The bottom of the waveguide is steel, which provides good reflection at this interface $[R>0.8$ for all angles - see, e.g., Mayer (1963)]. The geometry of the waveguide provides an average of 12 distinguishable ultrasonic arrivals within a reverberation

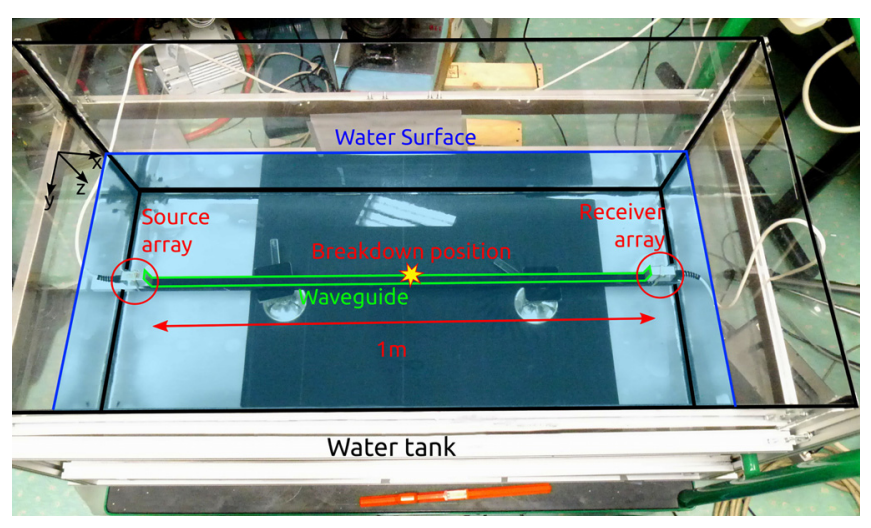

FIG. 1. (Color online) Annotated photograph of the experimental setup. The vertical 64-element source and receiver arrays face each other in a 1-mlong, 55-mm-deep water waveguide (highlighted in green). The waveguide dimensions are large compared to the $1.5-\mathrm{mm}$ wavelength of the ultrasonic wave. The bottom of the tank is made of steel, which allows for good energy reflection at this interface. The water surface (highlighted in blue) is perturbed by the laser-induced breakdown (yellow star) located up to $3.4 \mathrm{~cm}$ above the surface and centered between the source and receiver arrays. time window of $\approx 75 \mu$ s (i.e., about 75 times the duration of the emitted pulse).

Figure 2(a) shows the time-domain pressure field received by transducer $\sharp 33$ at a depth of $30 \mathrm{~mm}$ on the receiver array when the broadband pulse signal is emitted by transducer $\sharp 12$ at a depth of $14 \mathrm{~mm}$. Multiple echoes are clearly seen, which is expected in our reverberant medium.

The acquisition sequence consists of the recording of the pressure field for each source and each receiver in the time domain. A rapid way to perform this acquisition is by using a "round-robin" sequence, during which each source emits a broadband pulse successively [Roux and Nicolas (2014)]. The full waveguide transfer matrix is acquired every $10 \mathrm{~ms}$ for a total acquisition time of $5 \mathrm{~s}$, which provides 500 successive images. The waveguide perturbations are separately generated at the air-water interface by a laser-induced point-like source. As the frequency of the gravity-capillary surface wave is about $4.5 \mathrm{~Hz}$, the transfer matrix recording rate of $100 \mathrm{~Hz}$ ensures that the surface-wave perturbation is sampled correctly by the ultrasonic arrays. From here on, the total time of the experiment during which the surface is disturbed will be referred to as the "acquisition time" ( $5 \mathrm{~s})$, and the reverberation time window during which the pulse and its multiple echoes propagate through the waveguide will be referred to as the "propagation time" ( $\approx 75 \mu \mathrm{s})$.

The water-surface perturbation is caused by laserinduced breakdown (Fig. 3). The instant of the laser shot is referred to as the "temporal origin," and the acquisition chain that includes both the camera and the ultrasonic source-receiver arrays is triggered by a fast-response photodiode (Thorlabs SM1) that detects the laser beam during the shot. The laser source (pulsed Nd-YAG; Quanta Ray Pro; Spectra Physics) has a wavelength $\lambda=1064 \mathrm{~nm}$, and provides up to $3.5 \mathrm{~J}$ in a Gaussian-like temporal pulse of $9 \mathrm{~ns}$ width at half maximum. An optical circuit is used to guide the laser beam to a lens that focuses the energy at the desired position, where the breakdown occurs as the energy exceeds the breakdown energy. The breakdown causes a blast wave that propagates through the air and interacts with the water surface, to create the gravity-capillary wave ripples that are imaged. The breakdown is positioned between the emitting and the receiving arrays, and can be positioned either above the water surface or underwater. The ripples created by the breakdown propagate in all directions. However, given the planar configuration of the source-receiver array, the circular surface ripples are seen by the ultrasonic system as two counter-propagating wave packets that expand from the point origin.

To allow for independent measurement of the surface displacement, a high-speed camera (Photron SA2) records the side of the waveguide at 100000 frames/s, as shown in Fig. 4. A white back-lit screen is set up on the opposite side of the tank, to enhance the contrast of the surface perturbation. This camera provides an estimation of the water-surface displacement and the surface-wave group velocity. To remove the water meniscus that is formed at the tank wall and masks the water surface, a length of 25-mm-wide Teflon adhesive tape is positioned horizontally on the transparent wall in the water tank, along the water surface. The 

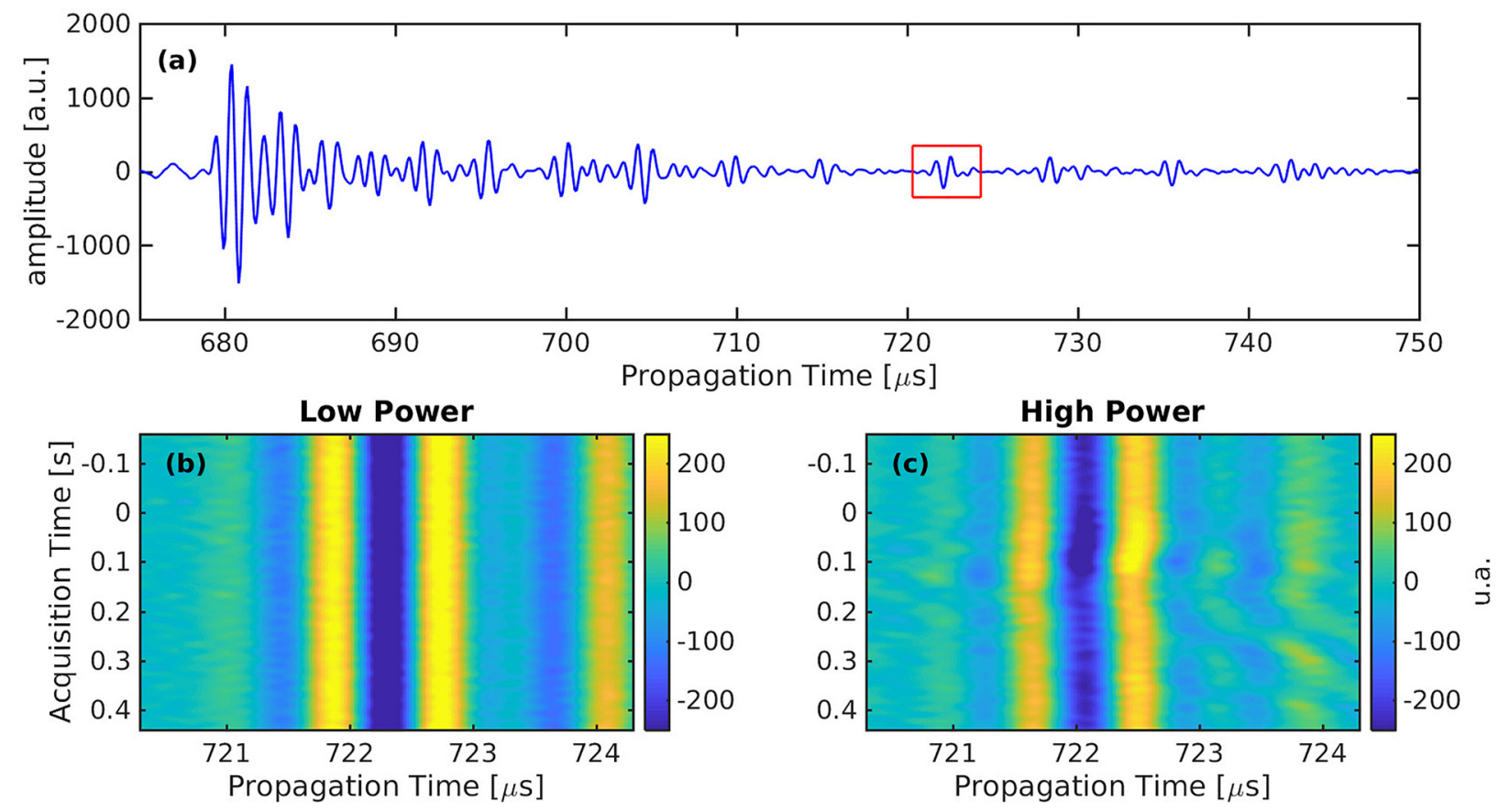

FIG. 2. (Color online) (a) Temporal signal recorded at transducer $\sharp 33$, at a depth of $z_{s}=30 \mathrm{~mm}$. The emitted signal is a 1 - $\mu$ s pulse that is emitted by transducer $\sharp 12$, at a depth of $z_{s}=14 \mathrm{~mm}$. The multi-reverberating behavior that defines the waveguide transfer function is clearly seen. The $x$ axis defines the propagation time, at $\mathrm{c}=1470 \mathrm{~m} / \mathrm{s}$ and $1-\mathrm{m}$ propagation distance. The $y$ axis defines the amplitude of the recorded signal. A weak signal is observed before the direct path (just before $680 \mu \mathrm{s}$ ) that is left over from the transmission from element $\sharp 11$ in the round-robin sequence. (b), (c) Enlargements of one of the ultrasonic arrivals, from the square in (a), for the "low-power" (b) and "high-power" (c) experiments. Travel-time and amplitude fluctuations are seen associated to the surface deformation after $\mathrm{T}=0 \mathrm{~s}$.

hydrophobic behavior of the Teflon tape removes the meniscus above the water surface, and makes it easier for the camera to monitor the water-surface deformation during the experiment. The laser-induced breakdown perturbation method allows good repeatability and control over both the localization and the intensity of the surface perturbation. Numerous surface excitations were performed throughout this study. In this paper, we present eight experimental

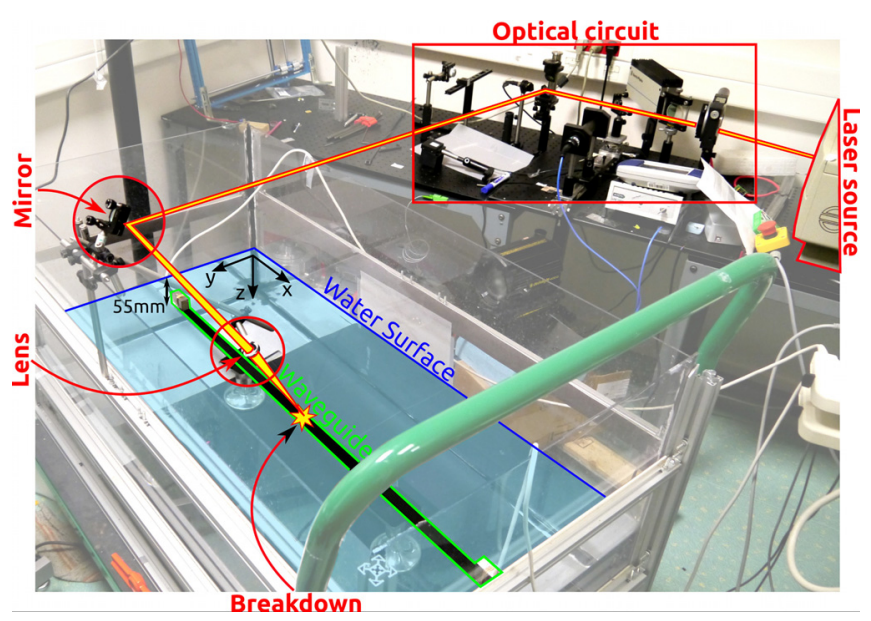

FIG. 3. (Color online) Annotated photograph of the experimental setup, with emphasis on the laser installation. The laser source is a Quanta Ray Pro from Spectra Physics, with a wavelength $\lambda=1064 \mathrm{~nm}$. The laser beam is guided using an optical circuit, to a lens that concentrates the energy at the desired position where the breakdown occurs as the energy reached exceeds the breakdown energy. The breakdown causes a shock-wave that propagates through air and interacts with the free surface, creating ripples at the air-water interface. datasets, with different energies and heights above the water surface. The extreme cases are emphasized, and are described as "low power" and "high power" throughout this paper. The low-power experiment refers to a laser shot at $30 \%$ full power with the breakdown position at $3.4 \mathrm{~cm}$ above the water surface; in the high-power experiment, the laser power is $95 \%$ and the breakdown position is $1 \mathrm{~cm}$ above the water surface. As breakdown closer to the surface will transfer more energy to the air-water interface than one further away, this provides a qualitative scale of excitation of the water surface, with the low-power experiment as weak surface perturbation, to the high-power experiment as strong surface perturbation.

When the water surface is disturbed, the pressure field recorded between the two arrays is modified. Following one

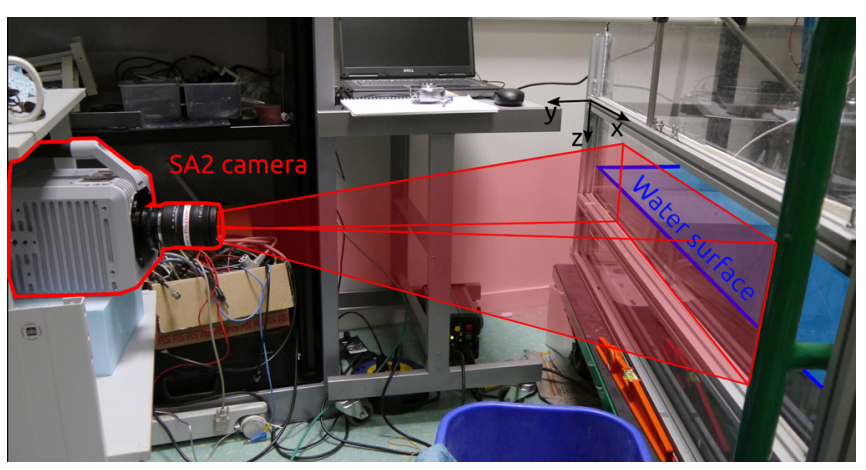

FIG. 4. (Color online) Annotated photograph of the high-frame SA2 camera that films from the side of the water waveguide (not seen here) at 100000 frames per second. This camera allows estimation of the water-surface displacement, the wave-group velocity, and the height of the laser breakdown. 
echo during the experiment, fluctuations in time and amplitude of the ultrasonic arrivals are observed [Figs. 2(b) and 2(c)]. These variations are important when the laser shot is very energetic, as shown in Fig. 2(c) at the acquisition time of $\approx 0.1 \mathrm{~s}$, although they are too subtle to be visible in the raw data for small surface perturbations, as seen in Fig. 2(b).

Using beam theory, the different arrivals of the multireverberated ultrasonic waves can be interpreted as individual eigenbeams of the waveguide. A double beamforming (DBF) algorithm, as described by Roux et al. (2008), allows the ultrasonic wavefield to be projected onto the waveguide eigenbeams, each of which is defined by its coordinates (emitting angle, receiving angle, travel-time). To extract the quantitative variations of each echo, DBF is performed on the source and receiver arrays, thus going from an emittingand-receiving sensor depth space $\left[z_{s}, z_{r}, t\right]$ to an emittingand-receiving angle space $\left[\theta_{s}, \theta_{r}, t\right]$. This representation allows the eigenray paths of the ultrasonic signal between each source and receiver to be isolated. As the eigenray concept results from the application of ray theory that is traditionally valid in an infinite bandwidth-high frequency approximation, in the following we prefer to describe the ultrasonic arrivals extracted from the DBF process as eigenbeams in the finite-frequency approach. Through DBF, the point-to-point pressure field $P\left(z_{s}, z_{r}, \omega\right)$ then becomes

$$
\begin{aligned}
P^{D B F}\left(\theta_{s}, \theta_{r}, t\right)= & \frac{1}{2 \pi} \frac{1}{N_{s}} \frac{1}{N_{r}} \sum_{s} \sum_{r} \int P\left(z_{s}, z_{r}, \omega\right) \\
& \times \exp \left[-i \omega\left(\tau\left(\theta_{s}, z_{s}\right)+\tau\left(\theta_{r}, z_{r}\right)\right)\right] \\
& \times \exp [-i \omega t] \mathrm{d} \omega,
\end{aligned}
$$

where $N_{s}$ the number of transducers in the source array, $N_{r}$ is the number of transducers in the receiver array, and $\theta_{s}$ and $\theta_{r}$ are the emitting and receiving angles of the eigenbeam, respectively. The time delays $\tau\left(\theta_{i}, z_{j}\right)$ for a wave emitted (or received) at an angle $\theta_{i}$ and for each element $j$ are defined as

$$
\tau\left(\theta_{i}, z_{j}\right)=\frac{\left(z_{j}-z_{0}\right) ; \sin \left(\theta_{i}\right)}{c},
$$

where $z_{0}$ is the depth of the center of the $N$-element array, and $c$ is the speed of sound.

In practice, the 64-element arrays are divided into subarrays on which the DBF is performed. In this experiment, 14 subarrays are used on each side, which provides $14 \times 14$ $=196$ source-receiver subarray pairs. We choose to work with eigenbeams that have emitting and receiving angles between 5 and $25 \mathrm{deg}$. The angular lower boundary $\left(5^{\circ}\right)$ is used to discard eigenbeams that cannot easily be individually recognized since early echoes are close to each other in the angle space. Also, the eigenbeam associated to a direct path does not interact with the free surface and does not provide any information for surface inversion. The upper angular boundary $\left(25^{\circ}\right)$ is defined to discard eigenbeams that have surface reflection that is too close to either of the arrays, as these do not respect the far-field hypothesis for the Green's function in the SK formulation, which also means that this approach performs badly in these regions. Within these bounds, it is possible to extract around 12 ray paths per source-receiver subarray pair. In total, we can use more than 2000 eigenbeams extracted by the DBF algorithm.

Following the same eigenbeam for the low-power and high-power experiments, the effects of the surface disturbances on the ultrasonic propagation are accurately monitored, and the two extreme experiments can be compared with each other. Figure 5 presents the normalized amplitude variation $\triangle A / A$ as a function of the acquisition time, for one eigenray that is selected through DBF for $\theta_{s}=19.6^{\circ}$ and $\theta_{r}=-19.3^{\circ}$. This corresponds to the ultrasonic arrival selected in Fig. 2. The difference between $\theta_{s}$ and $\theta_{r}$ is due to the slight horizontal slope of the bottom steel bar and the slight array tilt on either the source or receiver arrays (Roux and Nicolas, 2014). There are both quantitative and qualitative differences between the two DBF signals in Fig. 5. The high-power experiment not only shows the largest variations in amplitude $(\approx 10$ times larger than in the low-power experiment), but also nonlinear behavior right after the laser excitation (a slight negative "offset"), between $\mathrm{T}=0 \mathrm{~s}$ and $\mathrm{T}=0.5 \mathrm{~s}$, when the water surface perturbation is strongest. After $\mathrm{T}=0.5 \mathrm{~s}$, however, the linear regime is restored. We can also observe that the variations after $\mathrm{T}=0.5 \mathrm{~s}$ for both experiments are in phase, which indicates the same sensitivity of the eigenray to the surface waves, and therefore the repeatability of these experiments.

\section{THE SENSITIVITY KERNEL APPROACH}

The reflections at the surface of the set of eigenbeams cover the entire range $r^{\prime} \in[0,1] \mathrm{m}$ of the air-water interface between the source-receiver arrays. The use of SK theory

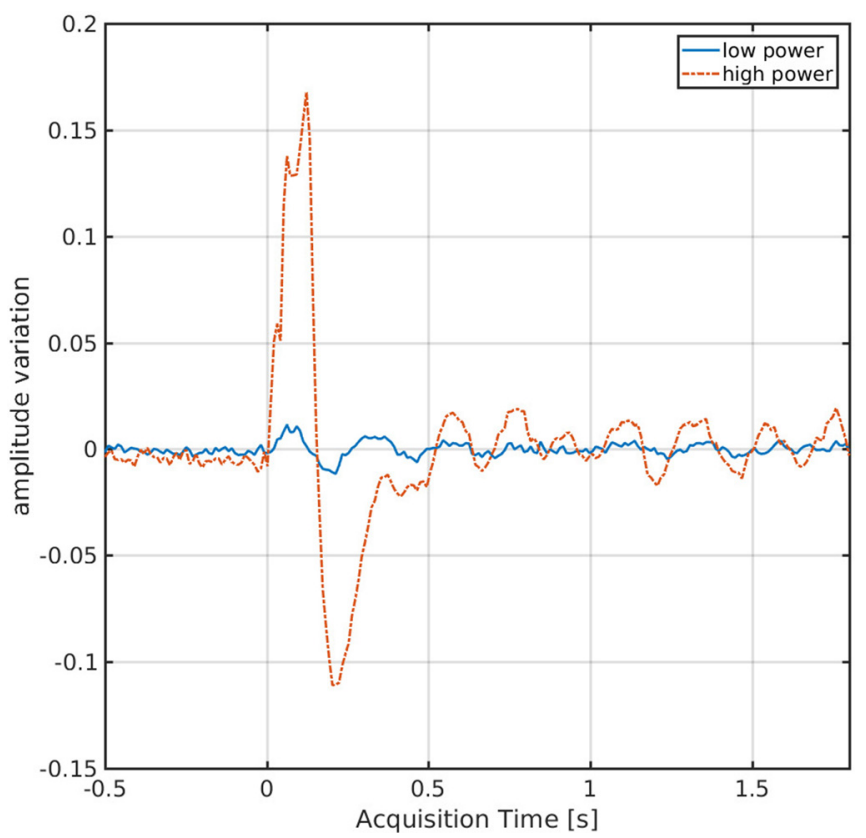

FIG. 5. (Color online) Amplitude variation $\Delta A / A$ extracted for one eigenbeam of the waveguide that corresponds to the wavelet plotted in Fig. 4, for the "low-power" (solid blue line) and "high-power" (dashed-dotted red line) experiments. The amplitude is the maximum of the output of the DBF algorithm, and the amplitude variation $\Delta A$ is normalized by the amplitude $A$ of the unperturbed eigenbeam. 
allows us to disregard travel-time information as a natural approach in acoustic tomography to retrieve the water-surface deformation, and instead to invert amplitude variations for the set of eigenbeams. The principle behind the SK approach is the linear relationship under the first-order Born approximation (Beydoun and Tarantola, 1988) between the water surface displacement $\Delta h$ at the surface of the waveguide at a range of $r^{\prime}$, and the normalized variation of the observable $\Delta A / A$ for an eigenbeam; i.e., the forward model is

$$
\frac{\Delta A\left(r^{\prime}, t\right)}{A}=\int_{\omega} \int_{r^{\prime}} K^{D B F}\left(\theta_{s}, \theta_{r}, r^{\prime}, \omega\right) \Delta h\left(r^{\prime}\right) \mathrm{dS} \mathrm{d} \omega,
$$

where $K^{D B F}$ is the amplitude SK for the selected eigenbeam (Sarkar et al., 2012). The approach used in this paper differs from Roux and Nicolas (2014), in that the SKs are computed for the entire surface and not only for the central line that joins the two arrays. This allows correct quantitative estimation of the height of the perturbation, as the whole ultrasonic beam is taken into account (with its lateral extent).

Note that the first-order Born approximation requires a small surface perturbation; i.e., a weak-amplitude surface wave. Equation (3) demonstrates that the amplitude variations of an eigenbeam are the linear summation of the elementary perturbations at all ranges, which are weighted according to the eigenbeam SK. The experiments reported in the present paper test these properties, as the two counterpropagative waves at either side of the central laser excitation source provide two distinct surface perturbations with different amplitudes.

For a source at $\mathbf{r}_{\mathrm{s}}$ and a receiver at $\mathbf{r}_{\mathbf{r}}$, the wave propagation in the unperturbed waveguide is given by the Green's function $G_{0}\left(\mathbf{r}_{\mathbf{s}}, \mathbf{r}_{\mathbf{r}}, \omega\right)$, where $\omega$ is the angular frequency. When the local perturbation $\Delta h$ is introduced in the onedimensional waveguide, the pressure field is modified by a small $\Delta p$. According to the definition of Sarkar et al. (2012), the expression for the point-to-point sensitivity kernel $K$ is

$$
\begin{aligned}
K\left(\mathbf{r}_{\mathbf{s}}, \mathbf{r}_{\mathbf{r}}, r^{\prime}, \omega\right) & =\frac{\Delta p\left(\mathbf{r}_{\mathbf{s}}, \mathbf{r}_{\mathbf{r}}, \omega\right)}{\Delta h\left(r^{\prime}\right)} \\
& =\frac{G\left(\mathbf{r}_{\mathbf{s}}, \mathbf{r}_{\mathbf{r}}, \omega\right)-G_{0}\left(\mathbf{r}_{\mathbf{s}}, \mathbf{r}_{\mathbf{r}}, \omega\right)}{\Delta h\left(r^{\prime}\right)}
\end{aligned}
$$

where $G\left(\mathbf{r}_{\mathbf{s}}, \mathbf{r}_{\mathbf{r}}, \omega\right)$ is the Green's function of the waveguide perturbed by the surface wave. Now, using Green's theorem and the first-order Born approximation, we can approximate the perturbed Green's function by

$$
\begin{aligned}
G\left(\mathbf{r}_{\mathbf{s}}, \mathbf{r}_{\mathbf{r}}, \omega\right) \approx & G_{0}\left(\mathbf{r}_{\mathbf{s}}, \mathbf{r}_{\mathbf{r}}, \omega\right)-\oint \nabla_{n} G_{0}\left(\mathbf{r}_{\mathbf{s}}, r^{\prime}, \omega\right) \\
& \times \Delta h\left(r^{\prime}\right) \nabla_{n} G_{0}\left(r^{\prime}, \mathbf{r}_{\mathbf{r}}, \omega\right) \mathrm{d} S
\end{aligned}
$$

where $\nabla_{n}$ is defined as the gradient operator projected along a unitary vector normal to the unperturbed surface [Sarkar et al. (2012)]. Furthermore, the SK approach requires that we project the Green's function on the space of the individual eigenbeams. Again, this can be done as in Eq. (1), using the DBF algorithm

$$
G^{D B}\left(\theta_{i}, r^{\prime}, \omega\right)=\frac{1}{N_{i}} \sum_{i} G\left(\mathbf{r}_{\mathbf{i}}, r^{\prime}, \omega\right) \times \exp \left[-i \omega \tau_{i}\right]
$$

where $i$ is either the source or the receiver. These considerations allow Eq. (4) to be rewritten as

$$
\begin{aligned}
K^{D B F}\left(\theta_{s}, \theta_{r}, r^{\prime}, \omega\right)= & G_{0}^{B F}\left(r^{\prime}, \theta_{s}, \omega\right) G_{0}^{B F}\left(r^{\prime}, \theta_{r}, \omega\right) \\
& \times \frac{\omega^{2}}{c^{2}} \sin \left(\tilde{\theta}_{s}\right) \sin \left(\tilde{\theta}_{r}\right),
\end{aligned}
$$

where $\tilde{\theta}_{s}$ (respectively, $\tilde{\theta}_{r}$ ) represents the eigenray angle at the source array center (respectively, the receiver array center) (Sarkar et al., 2012). Finally, as shown by Marandet et al. (2011), the SK for the amplitude variations can be expressed as

$$
\frac{\Delta A\left(r^{\prime}, t\right)}{A}=\frac{\Delta p^{D B F}\left(\theta_{s}, \theta_{r}, t, r^{\prime}\right)}{p^{D B F}\left(\theta_{s}, \theta_{r}, t\right)} .
$$

An example of a SK associated with the eigenbeam amplitude fluctuations can be seen in Fig. 6(b), where it corresponds to the eigenbeam shown in Fig. 6(c). The SK is plotted for every point $r^{\prime}$ at the surface of the waveguide along the directions parallel (i.e., range) and perpendicular (i.e., width) to the waveguide axis. As expected, the sensitivity is null everywhere at the surface of the waveguide except around the three positions where the eigenbeam hits the surface. Note that the width of the SK increases to $2 \mathrm{~cm}$, in agreement with the lateral dimension of each transducer $(12 \mathrm{~mm})$ within the source-receiver arrays. Ray tracing of the equivalent eigenray [Fig. 6(c)] helps to visualize the geometry of the eigenbeam. In Fig. 6(c), the red lines are the surface (depth $=0 \mathrm{~m}$ ) and the bottom (depth $=0.05 \mathrm{~m}$ ) of the waveguide, and the red stars are the centers of the emitting and receiving subarrays.

The SKs set the linear forward model between the amplitude variations $\Delta A$ and the surface displacement $\Delta h$. Using matrix formulation and waveguide discretization, Eq. (3) can be rewritten as

$$
\frac{\Delta A}{A}=K^{D B F} \Delta h \mathrm{~d} S
$$

In the present case, we set up the inverse problem to retrieve the surface displacement $\Delta h$ using the amplitude variations of the eigenbeams. In the general case, the matrix $K^{D B F}$ does not have an inverse. We use the matrix regularization used in the MAP scheme described by, e.g., Beydoun and Tarantola (1988) and Roux and Nicolas (2014). Therefore, an estimation of the displaced surface $\widehat{\Delta h}$ can be written as

$$
\widehat{\Delta h}=\frac{1}{d S} ; C_{m} K^{T}\left(K C_{m} K^{T}+C_{d}\right)^{-1} \frac{\Delta A}{A},
$$

where $K=K^{D B F}$ for the sake of notation, ${ }^{T}$ is the matrix transpose operator, $C_{m}$ is the covariance matrix of the model, and $C_{d}$ is the covariance matrix of the data. For the sake of simplicity, the data misfits on the beam observables are considered to be independent, i.e., $C_{d}$ is diagonal and $C_{d}=\alpha \mathbb{I}$, where $\alpha$ is the data misfit for all of the beam observables, as estimated from the variations in the data when the system is 

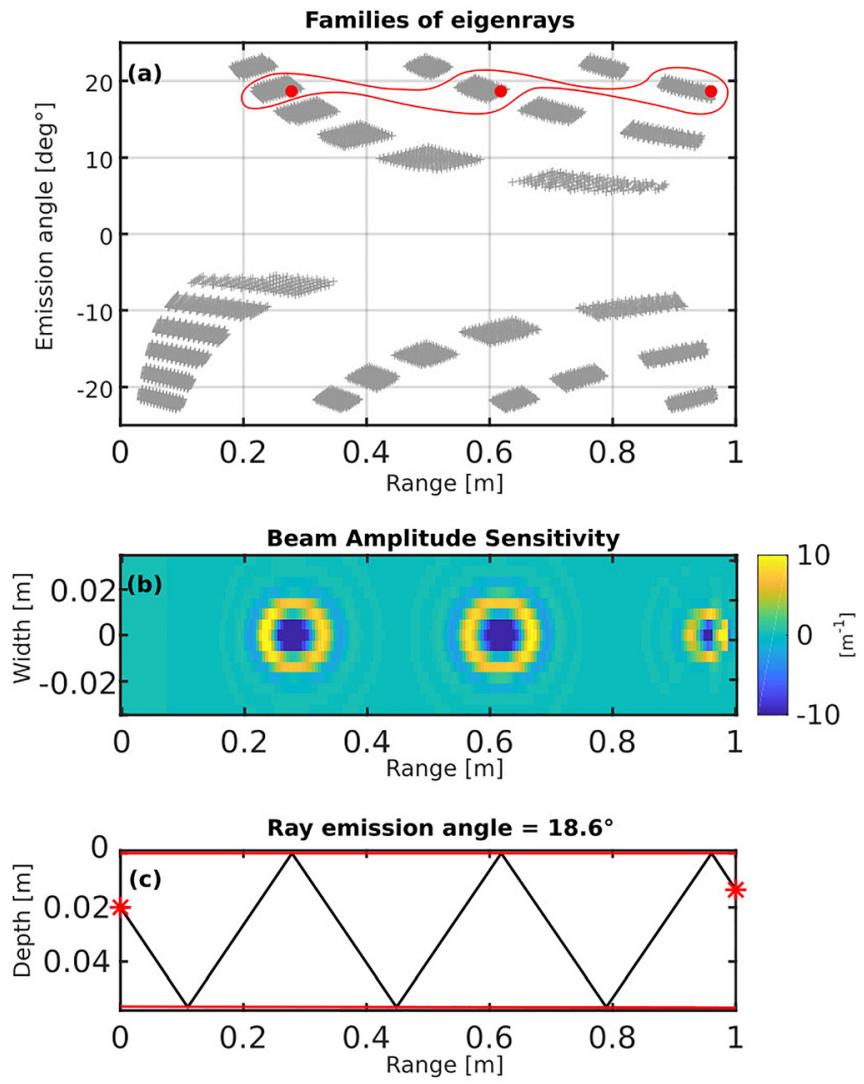

FIG. 6. (Color online) (a) Representation of all of the eigenrays of the waveguide, with their emission angle as a function of the position of their surface reflections (gray crosses). One eigenray hitting the surface three times is selected (red circles). (b) Surface Sensitivity for the amplitude variations associated with the waveguide eigenray selected in (a). (c) Raytracing of the waveguide eigenray selected in (a). Red lines, surface and bottom of the waveguide; red stars, centers of the emitting and receiving subarrays.

at rest. The model covariance matrix $C_{m}$ is set in such a way that reconstructed surface deformations are spatially correlated with a 2-cm smoothing distance (Roux and Nicolas, 2014).

\section{INVERSION RESULTS}

To perform good inversion, a solid dataset is needed. The data carry information in both time and space, because the fluctuations of each eigenbeam come from the water-surface displacement around the surface reflection points. Each eigenbeam images a small number of water-surface points in the inversion. It is thus necessary to work with a set of eigenbeams where the reflection points cover the whole range between the source and receiver arrays. Furthermore, redundancy within the eigenbeam set (due to the use of a large number of nearby subarray pairs) means that it is not necessary to include the whole set of eigenbeams in the inversion process. The scope of this study is not to discuss the critical number of independent eigenbeams that would optimize the inversion result, as this number might also depend on the regularization process used through the inversion procedure. However, some elements related to the eigenbeam redundancy are now discussed. Considering the emission angles and the positions of the eigenbeam surface reflections, different and disjoint eigenbeam families can be defined, as represented by the discrete patches in Fig. 6(a). Within each family, the eigenbeams have the same number of surface reflections, and are close to each other in terms of the emission and receiving angles.

On this basis, one beam is represented in Fig. 6(a) as many times as the number of times it hits the surface, as shown by the three red circles. The family corresponding to the selected eigenbeams is encircled, and in total, 12 distinct beam families can be discerned. It is interesting to discuss the discrete behavior of the distribution of eigenrays in this subspace. The discrete behavior in the emission angle dimension is ruled by the size of the receiving subarray. Continuity in the emission angle means an existing eigenray for each emitting angle, and therefore complete depth coverage by the receiving subarrays. The smaller the receiving subarrays, the closer their centers can go to the surface and bottom of the waveguide, and the smaller the gaps in the emission angle dimension. Note that the opposite works for the discrete behavior of the receiving angles (not shown here). Finally, the discrete behavior of the surface reflection position dimension (or range dimension) is ruled by the geometry of the waveguide, and in this case the depth-to-range ratio.

The inversion is performed on the whole surface of the waveguide, but for the sake of representation, the inversion result is shown as a function of the acquisition time and the waveguide range, as the width of the waveguide is much smaller than the range. Only the displacement at the central line of the surface (width $=0 \mathrm{~m}$ ) is therefore shown. Figure 7 compares the inversion results for low-power and high-power experiments. The surface displacement begins locally at range $=0 \mathrm{~m}$ and at time $=0 \mathrm{~s}$. As expected, stronger surface perturbation leads to larger water height displacement. The low-power experiment shows an estimate of the surface displacements $\widehat{\Delta h}$ of maximum $1 e-4 \mathrm{~m}$, while the high-power experiment shows a $\widehat{\Delta h}$ of maximum $2.5 e-3 \mathrm{~m}$. The dashed line in Figs. 7(a) and 7(b) highlights the group velocity. Note that for both experiments, the slope is the same, and it corresponds to a group velocity of $0.18 \mathrm{~m} / \mathrm{s}$.

Note that the low-power experiment indicates an inverted surface wave of under a tenth of a millimeter. The inversion result in the high-power experiment is about ten times larger, which is consistent with the ratio in the ray amplitude variations shown in Fig. 5. As the wavelength of the ultrasonic signal is $\lambda=1.5 \mathrm{~mm}$, we successfully image surface variations ranging from $\Delta h \approx \lambda / 10$ to $\Delta h \approx \lambda$. Furthermore, frequency-wavenumber $(\mathrm{F}-\mathrm{K})$ analysis of the acoustic inversion results (Fig. 11) shows an excellent match between the inversion results and the theoretical dispersion curve for gravity-capillary propagation, in agreement with a previous report (Roux and Nicolas, 2014).

In the following, we validate the ultrasonic inversion results with the independent optical measurements of the surface displacement. Figure 8 presents ten snapshots from the high-speed camera, with a resolution of $152 \times 256$ pixels and for the high-power experiment. In the first frame $(T=0 \mathrm{~s})$, the blast wave caused by the breakdown interacts with the surface of the water and creates a splash (frames one-two). If the splash is energetic enough, there is a Rayleigh jet (frames three-four) (for more details on the formation of Rayleigh jets, and higher definition images, see, e.g., Castillo-Orozco (2015). 

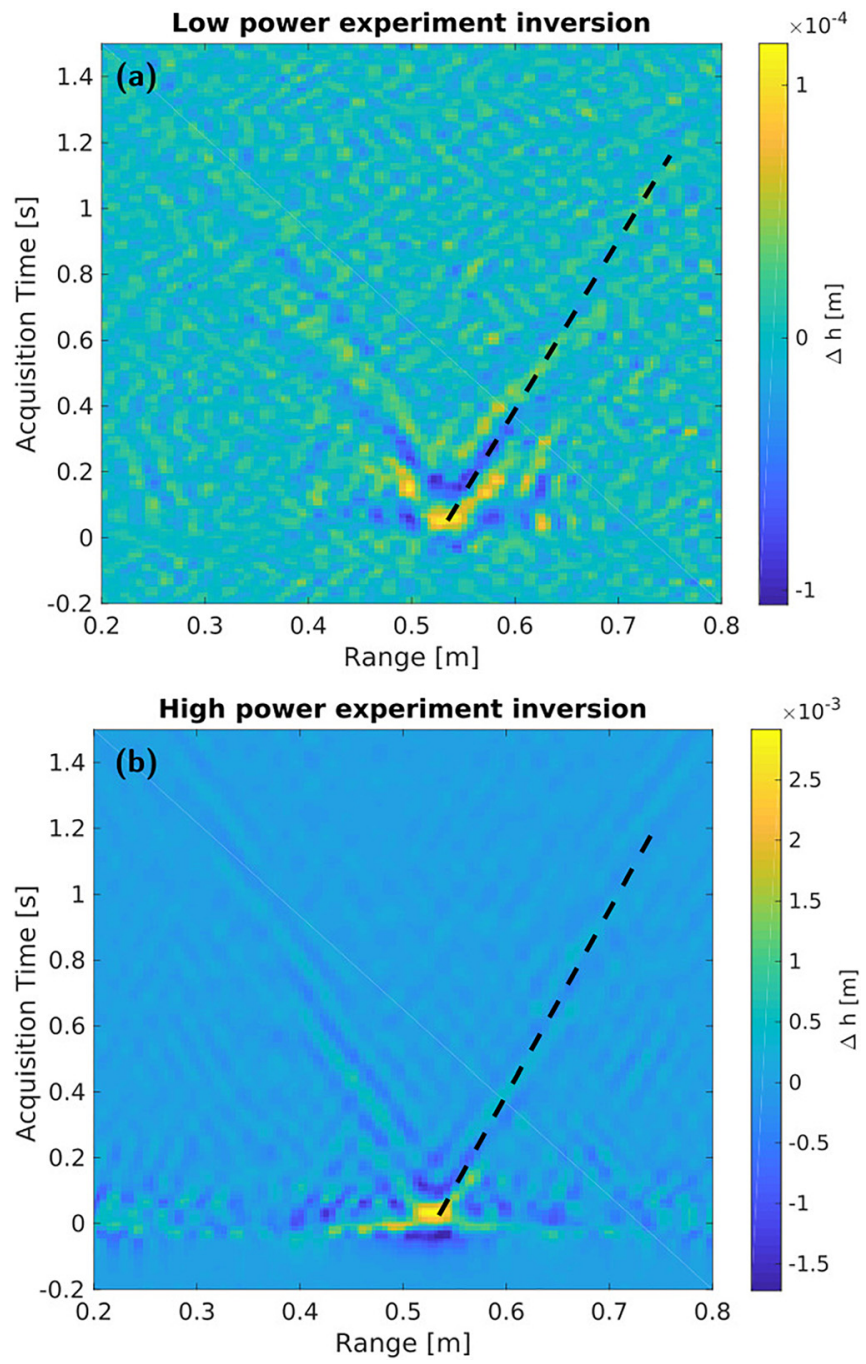

FIG. 7. (Color online) Inversion results for the "low-power" (a) and "highpower" (b) experiments, using the amplitude variation $\Delta A / A$. The $x$ axis is the length of the waveguide; the $y$ axis is the time relative to the laser shot; the color scale is the displaced water height. The color scale is different for each panel. The small negative displacement just before $\mathrm{T}=0 \mathrm{~s}$ is an artifact due to the temporal low-pass filtering. The dashed line highlights the group velocity $0.18 \mathrm{~m} / \mathrm{s}$.
Once the jet has fallen back, the water perturbation propagates and creates a gravity-capillary wave (frames five-nine). On the last frame ( $\mathrm{T}=0.23 \mathrm{~s}$ ) the wave is too small to be captured by the camera, which results in very bad signal-to-noise ratio. By analyzing the propagation of the perturbation in the camera frames, we can estimate the group velocity of $0.16 \mathrm{~m} / \mathrm{s}$, which is in agreement with the measurements taken from the ultrasonic inversion $(0.18 \mathrm{~m} / \mathrm{s})$.

These images also allow estimation of the height of the surface displacement. Considering the low resolution of the camera frames and the recording technique, only an orderof-magnitude estimate of the water height can be extracted. Also, the camera can capture surface disturbances of about a millimeter high or more. Therefore, only the strong experiments provide an optical measurement of the water height. Figure 9 shows the decrease in the envelope of the two ultrasonic inversions of the low-power and high-power experiments as a function of distance from the perturbation, on a $\log -\log$ scale. Indeed, the height of the surface displacement is a decreasing power law with the distance, which is consistent with the $1 / R$ law for the decrease of a two-dimensional wave. In addition, the average over the eight experiments with different laser excitation is also shown. Finally, the camera estimation of the water height displacement is plotted for the high-power experiment.

All-in-all, we get good agreement between the ultrasonic inversion of the high-power experiment and the optical estimation of the water surface displacement provided by the camera. The camera estimation starts off $\left(t<10^{-1} \mathrm{~s}\right)$ with the Rayleigh jet, which cannot be seen by the acoustic system. Once the Rayleigh jet has fallen back, the smallperturbation regime is restored and the camera estimation can be compared to the ultrasonic one. The value estimated by the camera confirms a wave of around $1 \mathrm{~mm}$ high at a distance of $1 \mathrm{~cm}$ from the perturbation. The camera estimation suddenly drops at around $2 \mathrm{~cm}$ from the perturbation, due to the limitations of the system for the capture of small surface displacements (Fig. 8). Furthermore, by confirming the inversion value of the high-power experiment, the camera
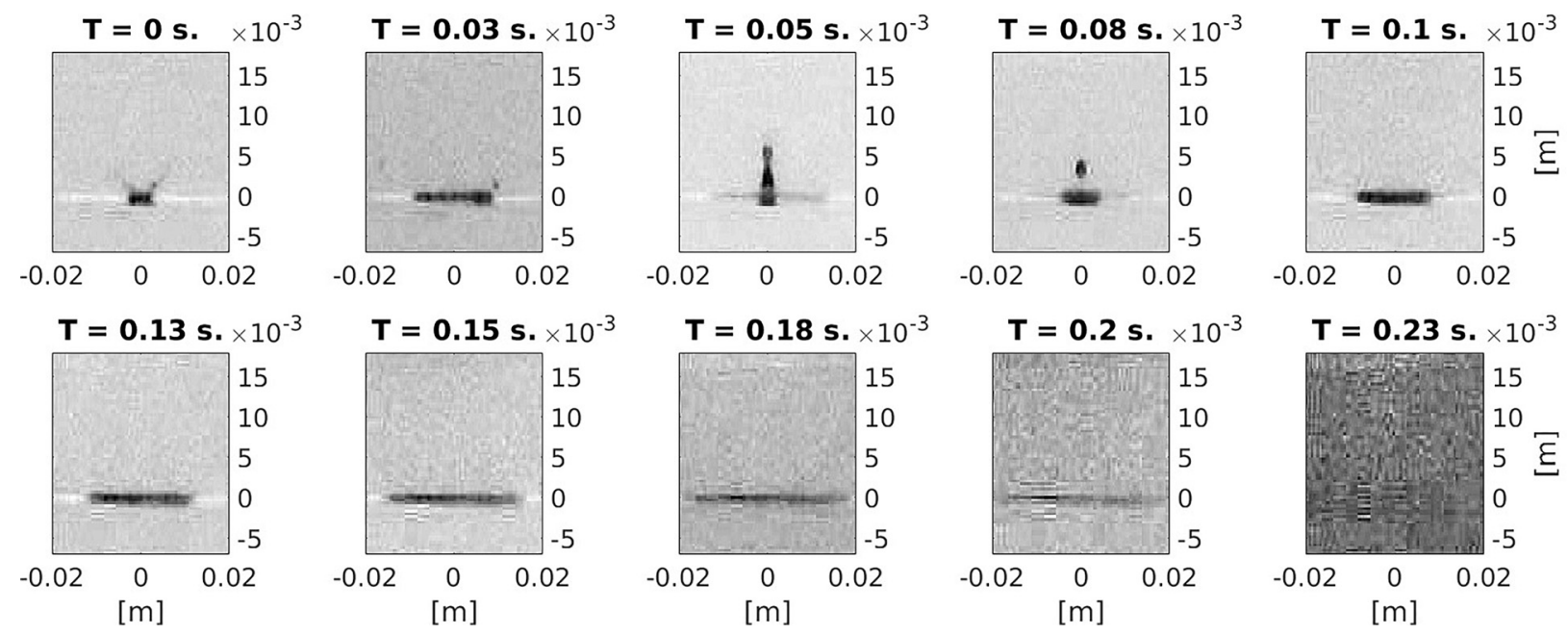

FIG. 8. Snapshots from the lateral high-speed camera that record the early times after the laser shot in the "high-power" experiment, showing the water-surface perturbation, the highly non-linear Rayleigh jet, and the surface-wave propagation from $\mathrm{T}=0.1 \mathrm{~s}$ on. The $x$ axis represents the distance relative to the center of the laser excitation, and the $y$ axis defines height relative to the water level. 


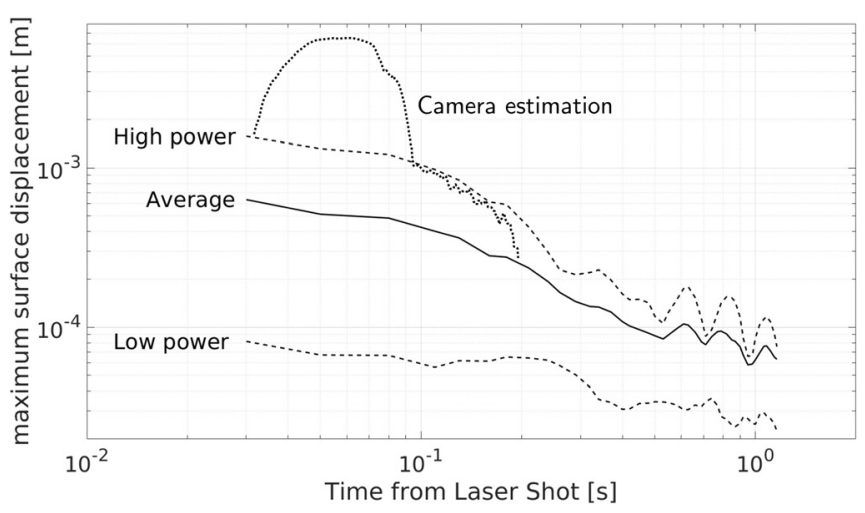

FIG. 9. Maximum of the surface wave as reconstructed using the acoustic system, as a function of its distance from the perturbation, plotted on a $\log -\log$ scale. Both the "low-power" and "high-power" experiments are represented as they show the extreme cases, and the average of the eight experiments is shown. The estimation of the water displacement for the high-power experiment provided by the camera recording is plotted in gray.

estimation also verifies the sub-millimeter inversion of the low-power experiment, as we have already shown that the tenfold ratio between the inversions of the low-power and high-power experiments is legitimate.

Last but not least, the maximum of the ultrasonic inversion results of all of the experiments are plotted in Fig. 10 as a function of the intensity of the blast wave created by the laser breakdown. The energy of the laser shot is an input parameter of the experiment, as is the height of the breakdown above the surface. We observe that the intensity of the blast wave when it interacts with the air-water interface is directly and linearly linked to the height of the surface wave. Again, the low-power experiment shows the smallest surface perturbation because the laser shot was relatively weak and was far above the surface, whereas the laser-induced blast wave in the high-power experiment has a much higher intensity when it hits the water surface.

\section{DISCUSSION}

The present study investigates dynamic imaging of gravity-capillary surface wave propagation using amplitude

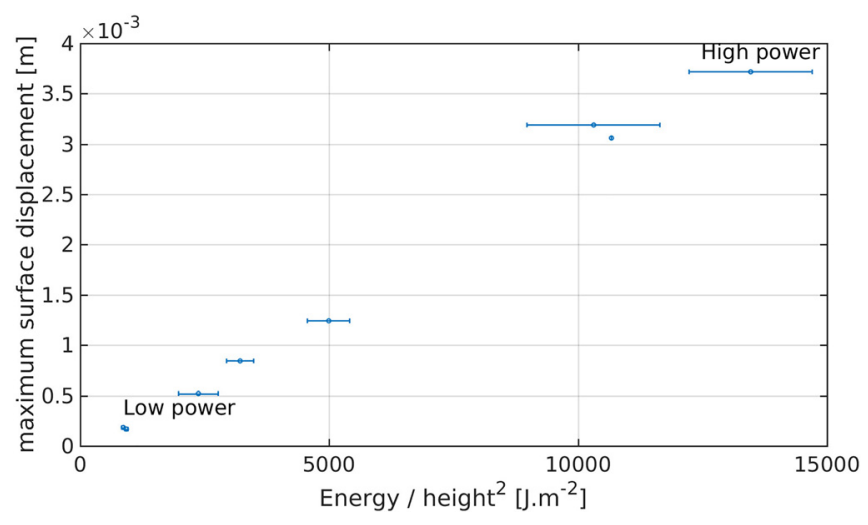

FIG. 10. (Color online) Acoustic inversion of the maximum of the surface displacement, as a function of the energy of the laser shot divided by the square of the height of the laser breakdown above the water surface. The low-power and high-power experiments show the extreme cases. The major contribution to the error bars is the uncertainty of the position of the laser breakdown. variations of waveguide eigenbeams. This imaging process is built using the ultrasonic wavefield variations between two transducer arrays that face each other in a waveguide. Independent measurements using an optical camera confirm the results of the inversion problem regarding the group velocity and surface displacement magnitude, and they also indicate the limitations that must be taken into account.

The main limitation of this inversion problem is the validity of the perturbation approach in the framework of the first Born approximation. This issue is well shown by the snapshots of the lateral high-speed camera, in Fig. 8, frames three and four. The splash induced by the laser-induced blast wave was energetic enough to cause a Rayleigh jet (frame three) which even breaks up into droplets (frame four). This highly nonlinear behavior falls beyond the small perturbation framework required by the SK approach. The high-speed camera is therefore very helpful to understand this physical problem, and to set the surface perturbation bounds within which to operate. However, the propagation of a gravity-capillary wave is a small surface displacement, and its recording using the sideview camera proved to be difficult for weak perturbations (e.g., for the low-power experiment). This setup is widely used for surface-wave monitoring at the laboratory scale in water tanks (e.g., Senet et al., 1999; Rousseaux et al., 2010), but the resolution of the high-speed camera $(152 \times 256$ pixels $)$ did not provide an accurate estimate of the height of the submillimeter gravity-capillary wave. In Fig. 7(b), the height of the central inverted displacement at $\mathrm{T}=0 \mathrm{~s}$ can therefore not be linked to any physical water-surface displacement. However, after $\mathrm{T}=0.1 \mathrm{~s}$, as the small-perturbation condition is again fulfilled, the inversion gives the corrected estimation for the water-surface displacement, as shown in Fig. 9.

Furthermore, in Fig. 7(b) we can observe that the inversion process allocates a surface displacement to the entire range at $\mathrm{T}=0 \mathrm{~s}$. This is a side-effect of the laser-induced blast wave. When it is very energetic (e.g., for the high-power experiment), it excites the steel bar that lies at the bottom of the waveguide. The vibrating bar then generates small ripples at the surface of the water, which are seen by the ultrasonic system. In the inversion result of the high-power experiment, after $\mathrm{T}=0.2 \mathrm{~s}$, the bar perturbation fades out and we are left with the image of the surface-wave propagation.

Within these limitations, this experiment succeeds in linking the water surface displacement and the strength of the perturbation. Figure 10 shows a clear linear trend between the surface displacement and the intensity of the blast wave that hits the surface. Again, this is not taking into account the nonlinear Rayleigh jet and the splashes, as these cannot be imaged by the ultrasonic system. This linear trend between the inversion result and the excitation parameter points to correct inversion of the water-surface displacement height.

\section{CONCLUSION}

This study uses amplitude fluctuations of waveguide eigenbeams, the inversion of which leads to accurate imaging of the propagation of a surface gravity-capillary wave. Through the use of SK formalism, two counter-propagating wave packets can be followed at the same time in two 


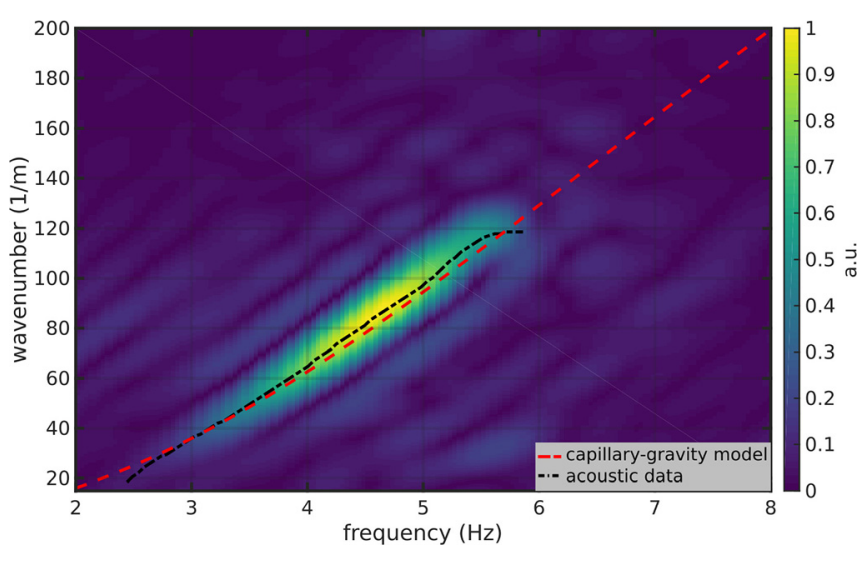

FIG. 11. (Color online) Modulus of the normalized frequency-wavenumber (F-K) transform of the ultrasonic inversion. The dashed-dotted line corresponds to the maxima of the F-K transform of the ultrasonic inversion; the dashed line corresponds to the theoretical capillary-gravity wave dispersion curve. This figure is provided for comparison with Fig. 10 from Roux and Nicolas (2014).

different places of the waveguide. The F-K spectrum of the surface deformation follows the theoretical dispersion curve of a gravity-capillary surface wave. Furthermore, an estimation of the height of the surface perturbation can be provided. This result is new, and it answers the discussions and limitations of the experiment by Roux and Nicolas (2014), which did not take into account the lateral extent of the SK, and therefore could not provide a correct water height estimate. Independent optical measurement of the surface perturbation is used to validate the ultrasonic inversion results. This confirms the accurate reconstruction of a gravity-capillary surface wave and the quantitative height estimation, as well as the limitations concerning the experimental setup and methodology. First, we observe that when surface perturbations are too strong, they cannot be imaged correctly using the SK formalism, as they fall outside the first-order Born approximation. Second, the optical system was not sensitive enough to capture the very small perturbations encountered in this experiment $(\Delta h \approx 1 e-4 \mathrm{~m})$, and can therefore only confirm the inversion results for the stronger perturbations. Future studies are planned to use variations in the emission and reception angles of the eigenbeams to perform the surface-wave inversion within the same SK approach. This new data analysis will complete the global picture defined by DBF of an ultrasonic wavefield in a waveguide.

\section{ACKNOWLEDGMENTS}

This study was supported by Direction Générale de l'Armement (DGA). ISTerre is part of Labex OSUG@2020. This work was performed within the framework of the LABEX Celya (ANR-10-LABX-0060) of Université de Lyon, within the program "Inverstissement d'Avenir" (ANR-16-IDEX-0005) operated by the French National Research Agency (ANR).

\section{APPENDIX}

\section{See Fig. 11.}

Beydoun, W. B., and Tarantola, A. (1988). "First Born and Rytov approximations: Modeling and inversion conditions in a canonical example," J. Acoust. Soc. Am. 83, 1045-1055.

Castillo-Orozco, E., Davanlou, A., Choudhury, P. K., and Kumar, R. (2015). "Droplet impact on deep liquid pools: Rayleigh jet to formation of secondary droplets," Phys. Rev. E 92, 053022.

Deane, G., Presig, J., Tindle, C., Lavery, A., and Stokes, M. (2012). "Deterministic forward scatter from surface gravity waves," J. Acoust. Soc. Am. 132, 3673.

Kuperman, W. A., and Lynch, J. (2004). "Shallow-water acoustics," Phys. Today 2004, 55-60.

Marandet, C., Roux, P., Nicolas, B., and Mars, J. (2011). "Target detection and localization in shallow water: An experimental demonstration of the acoustic barrier problem at the laboratory scale," J. Acoust. Soc. Am. 129, 85-97.

Mayer, W. G. (1963). "Reflection and refraction of mechanical waves at solid-liquid boundaries,” J. Appl. Phys. 34, 909-911.

Rousseaux, G., Maïssa, P., Mathis, C., Coullet, P., Philbin, T., and Leonhardt, U. (2010). "Horizon effect with surface waves on moving water," New J. Phys. 12, 095018.

Roux, P., Cornuelle, B. D., Kuperman, W. A., and Hodgkiss, W. S. (2008). "The structure of raylike arrivals in a shallow-water waveguide," J. Acoust. Soc. Am. 124, 3430.

Roux, P., and Nicolas, B. (2014). "Inverting for a deterministic surface gravity wave using the sensitivity-kernel approach,” J. Acoust. Soc. Am. 135, 1789.

Sarkar, J., Marandet, C., Roux, P., Walker, S., Cornuelle, B. D., and Kuperman, W. A. (2012). "Sensitivity kernel for surface scattering in a waveguide," J. Acoust. Soc. Am. 131, 111.

Senet, C., Braun, N., Lange, P., Seemann, J., Dankert, H., and Ziemer, F. (1999). "Image sequence analysis of water surface waves in a hydraulic wind wave tank," in Algorithms, Devices, and Systems for Optical Information Processing III, Denver, CO.

Trevorrow, M. (1998). "Boundary scattering limitations to fish detection in shallow waters," Fish. Res. 35, 127-135.

Walstead, S., and Deane, G. (2014). "Reconstructing surface wave profiles from reflected acoustic pulses using multiple receivers," J. Acoust. Soc. Am. 136, 604. 